\title{
Costos directos e impacto sobre la morbimortalidad hospitalaria de eventos adversos prevenibles a medicamentos en una institución de tercer nivel de Bogotá
}

\author{
José Fernando Pinzón¹, Carlos Maldonado¹, Jorge A. Díaz¹, Omar Segura²,3 \\ ${ }^{1}$ Departamento de Farmacia, Facultad de Ciencias, Universidad Nacional de Colombia, Bogotá, D.C., Colombia \\ 2 Posgrado de Gerencia de la Salud, Facultad de Enfermería, Fundación Universitaria de Ciencias de la Salud, \\ Bogotá, D.C., Colombia \\ ${ }^{3}$ Unidad de Investigaciones, Segura, Morón \& Castañeda Asesores en Salud, Ltda., Bogotá, D.C., Colombia \\ Lugar donde se realizó la investigación: Departamento de Farmacia, Facultad de Ciencias, Universidad Nacional \\ de Colombia, Bogotá, D.C., Colombia; y Hospital Universitario La Samaritana, Bogotá, D.C., Colombia
}

Introducción. La implementación de actividades de farmacovigilancia permite supervisar y evaluar aspectos relacionados con la atención médica. Es necesario que la información recolectada permita identificar oportunidades para mejorar la calidad de la atención en salud. Se propone un análisis de los eventos adversos a medicamentos desde la óptica preventiva y económica, estableciendo su impacto local.

Objetivo. Determinar el porcentaje de eventos adversos prevenibles a medicamentos, reportados en una institución de tercer nivel, para establecer su impacto sobre la morbimortalidad y el económico desde la óptica del pagador y mostrando la relevancia de usar un método que permita identificarlos y evitar o disminuir su presentación.

Materiales y métodos. A partir de los reportes generados durante actividades de farmacovigilancia en el año 2007 en un hospital de tercer nivel de Bogotá, se revisaron las historias clínicas de los pacientes involucrados y se evaluó la relación de causalidad, la gravedad y el carácter prevenible de los eventos adversos a medicamentos. Se calcularon los costos directos generados, agrupándolos en pruebas diagnósticas, tiempo de estancia adicional, procedimientos y medicamentos adicionales.

Resultados. Se revisaron 448 reportes de eventos adversos a medicamentos en 283 pacientes y se encontró que $24,8 \%$ de los eventos reportados eran prevenibles, con mortalidad de $1,1 \%$ y costos totales asociados con su atención entre \$33’620.346 (US\$16.687) y \$37’754.856 (US\$18.739). Los factores más frecuentemente asociados con la prevención fueron las interacciones farmacológicas y las dosis o frecuencias inadecuadas de administración.

Conclusiones. Es importante tomar medidas tendientes a disminuir la presentación de eventos adversos prevenibles a medicamentos, ya que repercuten negativamente tanto sobre la salud de los pacientes, como en el consumo de recursos.

Palabras clave: toxicidad de medicamentos, hospitales, costos y análisis de costo, estudios de evaluación, Colombia.

\section{Direct costs and hospital morbimortality impact from preventable adverse drug events}

Introduction. Implementing pharmacovigilance activities consists of monitoring and assessment of activities related to medical attention. However, additional data are necessary to identify conditions where care quality can be improved. Therefore, a focus on adverse drug events analysis from a prevention and economic perspective is needed, with emphasis on its local impact.

Objective. Preventable adverse drug events were summarized to establishing their impact on morbidity and mortality, as well as to estimate the ensuing economic burden.

Materials and methods. The data were gathered from a level 3 hospital (high complexity), located in Bogotá, Colombia, where specific pharmacovigilance activities were recorded in 2007. Patient charts were reviewed to characterize adverse drug events according to their causality, severity and preventability. Direct costs were estimated by grouping diagnostic tests, length of hospitalization, procedures and additional drugs required.

Results. The charts of 283 patients and 448 reports were analyzed. These data indicated that $24.8 \%$ of adverse drug events were preventable and that an associated mortality of $1.1 \%$ had occurred. The associated direct costs were between USD $\$ 16,687$ and $\$ 18,739$. Factors more commonly associated with preventability were drug-drug interactions, as well as inappropriate doses and unsuitable frequencies at which the drugs were administrated. 
Conclusions. The data recommended that actions be taken to decrease preventable adverse drug events, because of negative impact on patient's health, and unnecessary consumption of healthcare resources.

Key words: Drug toxicity, hospitals, costs and cost analysis, evaluation studies, Colombia.

Las reacciones secundarias y los eventos adversos a medicamentos han sido reconocidos como una de las categorías más importantes de enfermedad iatrogénica en términos de morbilidad y mortalidad, así como por sus implicaciones éticas y económicas (1). En el estudio Harvard Medical Practice se encontró que una de las mayores causas de efectos nocivos relacionados con la atención médica, era el uso de medicamentos (2). Se estima que, en los países industrializados, las reacciones adversas a medicamentos se asocian con 5 a $10 \%$ del total de los costos hospitalarios anuales (3).

La tasa de incidencia de las reacciones adversas a medicamentos presenta cifras variables entre $1,5 \mathrm{y}$ $35 \%$ en pacientes hospitalizados, dependiendo del método de detección usado (4), con una mortalidad de $0,32 \%$ (5) y un porcentaje de reacciones adversas prevenibles que fluctúa entre 18,7 y $73,2 \%$ (6). El establecimiento de una conexión de causalidad con el medicamento es fundamental para el entendimiento de la reacción adversa y, en ese sentido, la farmacovigilancia brinda herramientas para evaluar la causalidad entre el medicamento y el evento adverso, en forma individual (7).

Schumock y Thornton consideran que el fin último de los programas de farmacovigilancia es mejorar la seguridad y el uso racional de los medicamentos y, por ende, mejorar la atención del paciente y la salud pública (1). En este sentido, propusieron un esquema de siete preguntas orientadas a establecer si las reacciones adversas a medicamentos podían ser prevenidas o no. La evaluación de los reportes de reacciones adversas a medicamentos desde la perspectiva de la prevención, permite identificar áreas neurálgicas que pueden intervenirse con el fin de reducir la incidencia de dichas reacciones, lo cual produce un impacto positivo en las cifras de morbilidad y mortalidad generadas por esta causa $y$, a su vez, se traduce en un ahorro de recursos dentro del sistema de salud (8).

Teniendo en cuenta que en nuestro medio no se han llevado a cabo estudios de este tipo, existe la

Correspondencia:

José Fernando Pinzón, Calle 20C № 93-25, interior 4, apartamento 302, Bogotá, D.C., Colombia

Teléfono: (571) 266 1846; fax: (571) 654 4444, extensión 3999 ferpin06@yahoo.com

Recibido: 15/12/10; aceptado:30/03/11 necesidad de realizar uno enfocado en el análisis de reacciones prevenibles, estableciendo su impacto en el contexto local. Por ello, se propuso analizar los eventos adversos a medicamentos, reportados en una institución de tercer nivel: estableciendo el porcentaje de eventos prevenibles y su impacto sobre la morbimortalidad hospitalaria, caracterizando los eventos reportados, teniendo en cuenta aspectos propios de los pacientes, de los grupos de medicamentos implicados, así como su clasificación según su nivel de causalidad y gravedad, y proponiendo acciones específicas tendientes a disminuir los casos prevenibles. También, desde el punto de vista económico y bajo la óptica del pagador, entendiéndose como tal las Entidades Promotoras de Salud (EPS) y, en algunos casos, las aseguradoras que cubren el Seguro Obligatorio de Accidentes de Tránsito (SOAT), se propuso mostrar la relevancia que tiene la utilización de un método que permita identificarlos y evitar o disminuir su presentación.

\section{Materiales y métodos}

Se hizo un estudio fármaco-epidemiológico, descriptivo y retrospectivo, en el que se analizaron los reportes producidos como resultado de las actividades de farmacovigilancia del Hospital Universitario La Samaritana de Bogotá, durante el año 2007, obtenidos por notificación espontánea durante todo el año o por actividades específicas de vigilancia, producto del proyecto WHEDIT (Warfarina, Heparinas, Dipirona, Tramadol), consistente en la detección activa de eventos adversos sospechosos de ser producidos por estos medicamentos en pacientes hospitalizados, el cual se ejecutó durante los meses de marzo a agosto de $2007(9,10)$. Igualmente, se agregaron los casos detectados durante la revisión de las historias clínicas de los pacientes reportados. Se excluyeron los reportes de pacientes en quienes el evento fue la causa de ingreso hospitalario.

Se registraron los datos relevantes de los pacientes como sexo, edad, número de medicamentos recibidos, diagnósticos de ingreso y egreso, nivel de escolaridad y ocupación.

Como evento adverso a medicamentos se definió cualquier suceso médico desafortunado que puede presentarse durante un tratamiento con un medicamento, pero que no tiene necesariamente 
relación causal con el mismo (11). Como reacción adversa a medicamentos se consideró la definición propuesta por la Organización Mundial de la Salud (OMS), a saber: respuesta que es perjudicial e indeseada, que ocurre a dosis normalmente usadas en humanos para profilaxis, diagnóstico o tratamiento de una enfermedad, o para la restauración, corrección o modificación de una función fisiológica (12).

Para establecer la relación de causalidad, se usaron los criterios de la OMS (13). La gravedad se determinó según la escala de Hartwig y Siegel (cuadro 1) (14) y se identificaron los eventos adversos prevenibles a medicamentos según los criterios de Schumock y Thornton (1). Los casos se analizaron según el juicio clínico del investigador, apoyado en la historia clínica de cada paciente. Cuando se consideró necesario, se discutió con un par evaluador para lograr consenso sobre tópicos específicos.

El registro de los eventos adversos a medicamentos reportados se hizo utilizando el diccionario WHOART (World Health Organization Adverse Reaction Terminology). Se llevó a cabo un subanálisis tomando únicamente los casos con relación de causalidad definitiva o probable, los cuales se consideraron como reacciones adversas a medicamentos (15).

Se identificaron los costos directos generados por eventos adversos a medicamentos desde la óptica del pagador y se agruparon en cuatro categorías: pruebas diagnósticas, interconsultas requeridas, días adicionales de estancia hospitalaria y tratamientos adicionales, divididos en procedimientos y medicamentos adicionales requeridos como consecuencia del evento, de manera que cada evento se consideraría como una unidad de costo. Para cuantificar los costos por procedimientos, pruebas diagnósticas, valoraciones médicas y estancia hospitalaria, se utilizó como referencia el manual tarifario SOAT vigente al año 2007. En el caso de los medicamentos, se tomaron cuatro escalas: una con los valores facturados por la institución, y otras tres definidas como valor máximo, valor mínimo y la mediana de los precios para cada medicamento de la revista Farmaprecios (edición de diciembre de 2007). Para la conversión a moneda extranjera (dólares), se aplicó la tasa representativa del mercado a diciembre 31 de 2007 $(\$ 2.014,76)(16)$.

La información se procesó con el paquete estadístico SPSS $\AA_{\text {, }}$ versión 16.0 (versión de
Cuadro 1. Escala de evaluación de gravedad de eventos adversos a medicamentos (14)

Nivel 1: se presentan eventos adversos, pero no se requiere cambio en el tratamiento con el medicamento sospechoso.

Nivel 2: el evento adverso requiere que el medicamento sospechoso sea retirado, descontinuado o cambiado. No se requiere antídoto $u$ otro tratamiento, y no hay aumento del tiempo de estancia hospitalaria.

Nivel 3: el evento adverso obliga a que el medicamento sea suspendido, descontinuado o cambiado, y se requiere un antídoto $u$ otro tratamiento. No hay aumento del tiempo de estancia hospitalaria.

Nivel 4: (a) cualquier evento adverso a medicamentos de nivel 3 que incrementa el tiempo de estancia hospitalaria por al menos un día, o (b) el evento adverso es la razón de la admisión.

Nivel 5: cualquier evento adverso a medicamentos que requiere cuidado intensivo médico.

Nivel 6: el evento adverso causa daño permanente en el paciente.

Nivel 7: el evento adverso conduce directa o indirectamente a la muerte del paciente.

evaluación), haciendo un análisis descriptivo y un análisis bivariado de acuerdo con los eventos adversos a medicamentos clasificados como prevenibles y no prevenibles, utilizando la prueba de ji al cuadrado para las variables categóricas y la prueba t de Student para las continuas, con un 95 $\%$ de confianza. Se estableció un modelo de costos generados por eventos prevenibles, mediante el análisis de regresión lineal.

El proyecto recibió aprobación por parte del Comité de Ética del Hospital Universitario de La Samaritana.

\section{Resultados}

\section{Análisis de eventos adversos a medicamentos}

Se reportaron 456 eventos, de los cuales se excluyeron 19 por ser causa de ingreso o estar presentes al momento de la hospitalización. En total, se analizaron 448 casos en 283 pacientes hospitalizados en los servicios de medicina interna, ortopedia y cirugía, lo que corresponde a 2,98 \% del total de egresos durante el año 2007. De ellos, $415(92,6 \%)$ resultaron de la vigilancia intensiva del proyecto WHEDIT, 22 (4,9\%) se reportaron de manera espontánea a lo largo del año y, además, se agregaron $11(2,5 \%)$ casos encontrados en la revisión de las historias clínicas de los pacientes referidos en los reportes.

En lo referente al sexo, 48,9\% pertenecía al femenino y $51,1 \%$ al masculino. El promedio de edad general fue de 54,1 años (rango 15 a 93 años; 
$\left.I_{95 \%}: 52,8-55,4\right)$. El número de medicamentos recibidos simultáneamente al momento de presentarse el evento fue, en promedio, de 7,46 $\pm 2,93$ (rango, 2 a 18). Los datos que muestran la diferenciación entre casos prevenibles y no prevenibles, se explican en el cuadro 2.

Las reacciones que tuvieron un mayor porcentaje de eventos prevenibles fueron INR (International Normalized Ratio) aumentado (93,3 \%), INR disminuido (50\%), melenas (50\%) y erupciones cutáneas (40\%). El 68,3\% de los eventos asociados a warfarina, el $50 \%$ de los asociados a enoxaparina y el $2,4 \%$ de los eventos asociados a tramadol, se consideraron prevenibles. De acuerdo con la clasificación ATC (Anatomical, Therapeutic, Chemical Classification System), la mayoría de las sospechas de reacciones adversas se asociaron a medicamentos que actúan sobre la sangre y los órganos hematopoyéticos (48,4\%) y a analgésicos (45,1\%). A su vez, el 32,3\% de eventos adversos asociados a medicamentos que actúan en sangre y el $42,9 \%$ asociado a diuréticos, se consideraron prevenibles.

Se consideraron como factores asociados a la prevención, las respuestas afirmativas a las preguntas propuestas por Schumock y Thornton (cuadro 3) (1), y se encontró que el mayor número está asociado a la presencia de interacciones medicamentosas ( $45 \%$ ) y a dosis, ruta o frecuencia de administración no apropiadas para la edad, el peso o el estado de la enfermedad $(51,4 \%)$.

\section{Análisis de reacciones adversas a medicamentos}

Tomando únicamente los casos con relación de causalidad probada o probable (129), las variables donde se encontró diferencia estadísticamente significativa entre eventos adversos prevenibles y no prevenibles, fueron: aumento de estancia hospitalaria, necesidad de procedimientos, interconsultas y pruebas diagnósticas adicionales, reacciones adversas a medicamentos asociadas a warfarina, evento serio y uso de más de siete medicamentos simultáneos, todas ellas con relación directa con los eventos adversos prevenibles. Las reacciones adversas relacionadas con tramadol mostraron una diferencia significativa, pero con una relación inversa (mayores casos no prevenibles). Otras variables analizadas en las cuales no se encontró diferencia, fueron: necesidad de medicamentos adicionales, relación con uso de dipirona, heparina no fraccionada y dalteparina,
Cuadro 2. Eventos adversos prevenibles a medicamentos y no prevenibles, categorizados por grupos de edad y variables de los eventos

\begin{tabular}{|c|c|c|}
\hline Variable & $\begin{array}{c}\text { Evento } \\
\text { prevenible } \\
n=111 \\
n(\%)\end{array}$ & $\begin{array}{c}\text { Evento } \\
\text { no prevenible } \\
\mathrm{n}=337 \\
\mathrm{n}(\%)\end{array}$ \\
\hline \multicolumn{3}{|l|}{ Grupos de edad (años) } \\
\hline 15 a 35 & $27(26,7)$ & $74(73,3)$ \\
\hline 36 a 50 & $12(18,2)$ & $54(81,8)$ \\
\hline 51 a 65 & $16(14,8)$ & $92(85,2)$ \\
\hline 66 a 80 & $38(31,9)$ & $81(68,1)$ \\
\hline$>80$ & $18(33,3)$ & $36(66,7)$ \\
\hline \multicolumn{3}{|l|}{ Relación de causalidad } \\
\hline Definitiva & $21(38,9)$ & $33(61,1)$ \\
\hline Probable & $31(41,3)$ & $44(58,7)$ \\
\hline Posible & $43(21,9)$ & $153(78,1)$ \\
\hline Dudosa & $14(12)$ & $103(88)$ \\
\hline No clasificable & $2(33,3)$ & $4(66,7)$ \\
\hline \multicolumn{3}{|l|}{ Nivel de gravedad } \\
\hline 1 & $33(14,8)$ & $190(85,2)$ \\
\hline 2 & $20(31,7)$ & $43(68,3)$ \\
\hline 3 & $35(30,2)$ & $81(69,8)$ \\
\hline 4 & $17(54,8)$ & $14(45,2)$ \\
\hline 5 & $1(33,3)$ & $2(66,7)$ \\
\hline 6 & $0(0)$ & $1(100)$ \\
\hline 7 & $5(45,5)$ & $6(54,5$ \\
\hline \multicolumn{3}{|l|}{ Grupos ATC } \\
\hline $\begin{array}{l}\text { Sangre y órganos } \\
\text { hematopoyéticos }\end{array}$ & $74(32,3)$ & $156(67,8)$ \\
\hline Analgésicos & $36(16,8)$ & $178(83,2)$ \\
\hline Antibióticos sistémicos & $2(14,3)$ & $12(85,7)$ \\
\hline Diuréticos & $3(42,9)$ & $4(57,1)$ \\
\hline Anaerobicidas & $0(0)$ & $3(100)$ \\
\hline Sistema gastrointestinal & $0(0)$ & $3(100)$ \\
\hline Anticonvulsivos & $1(100)$ & $0(0)$ \\
\hline Corticosteroides sistémicos & $0(0)$ & $1(100)$ \\
\hline Soluciones electrolíticas & $1(100)$ & $0(0)$ \\
\hline Xantinas & $1(100)$ & $0(0)$ \\
\hline
\end{tabular}

ATC: Anatomical, Therapeutic, Chemical Classification System

sexo, presencia de más de un medicamento sospechoso y evento no reportado (cuadro 4).

\section{Análisis de costos}

En 283 casos $(63,2 \%)$ se generaron costos adicionales asociados a la presentación de eventos adversos a medicamentos. Los costos totales atribuibles a eventos prevenibles oscilaron entre $\$ 33^{\prime} 620,346$ (US\$16.687) y $\$ 37^{\prime} 754,855$ pesos (US\$18.739). Se encontró que 58,5 de los costos asociados a estancia hospitalaria adicional, $45,2 \%$ correspondientes a interconsultas y $37,5 \%$ asociados a pruebas diagnósticas, correspondieron a eventos adversos prevenibles (cuadro 5).

Tomando únicamente las reacciones adversas a medicamentos definitivas y probables, se encuentra 
Cuadro 3. Aplicación de preguntas para establecer eventos prevenibles

\begin{tabular}{lrr}
\hline Pregunta asociada a la prevención & Afirmativa & $\%$ \\
\hline 1. ¿El medicamento en cuestión no fue apropiado para la condición clínica del paciente? & 13 & 11,7 \\
2. ¿La dosis, ruta o frecuencia de administración, no fue apropiada para la edad, el peso o el estado & 57 & 51,4 \\
$\quad$ de la enfermedad? & 10 & 9,0 \\
3. ¿La supervisión terapéutica del medicamento u otras pruebas de laboratorio necesarias, & 1 & 0,9 \\
$\quad$ no se realizaron? & 50 & 0 \\
4. ¿Existía el antecedente de alergia o reacción adversa previa al medicamento? & 0,0 \\
5. ¿Estuvo implicada una interacción medicamentosa en la reacción? & 0 & 0,0 \\
6. ¿Se documentó una concentración sérica tóxica del fármaco? & 111 &
\end{tabular}

Adaptado de: Schumock GT, Thornton JP. Focusing on the preventability of adverse drug reactions. Hosp Pharm. 1992;27:538.

Cuadro 4. Cruce de variables significativas respecto a reacciones adversas a medicamentos prevenibles, con relación de causalidad definitiva o probable

\begin{tabular}{|c|c|c|c|c|c|c|}
\hline Variable & $\mathbf{n}$ & \multicolumn{2}{|c|}{ Prevenible } & $\%$ prevenible & Valor $p$ & $\begin{array}{c}\text { Relación } \\
\text { con prevenible }\end{array}$ \\
\hline \multicolumn{7}{|l|}{ Necesidad de aumento estancia } \\
\hline hospitalaria & 23 & 8 & 15 & 65,2 & 0,010 & Directa \\
\hline Evento serio & 69 & 29 & 40 & 58,0 & 0,000 & Directa \\
\hline \multicolumn{7}{|l|}{ Necesidad de pruebas } \\
\hline diagnósticas & 58 & 19 & 39 & 67,2 & 0,000 & Directa \\
\hline Asociado a tramadol & 35 & 35 & 0 & 0,0 & 0,000 & Indirecta \\
\hline Asociado a warfarina & 41 & 9 & 32 & 78,0 & 0,000 & Directa \\
\hline \multicolumn{7}{|l|}{ Necesidad de procedimientos } \\
\hline adicionales & 21 & 6 & 15 & 71,4 & 0,003 & Directa \\
\hline Necesidad de interconsultas & 39 & 17 & 22 & 56,4 & 0,019 & Directa \\
\hline $\begin{array}{l}\text { Menos de siete medicamentos } \\
\text { simultáneos }\end{array}$ & 61 & 45 & 16 & 26,2 & 0,002 & Indirecta \\
\hline
\end{tabular}

que los costos asociados a éstas oscilaron entre $12,8 \%$ (procedimientos) y 65,3 \% (estancia hospitalaria adicional) de los costos directos totales; en relación con la suma total de todos los eventos, este subconjunto representa entre 35,3 y $35,7 \%$, con valores que varían entre \$29'673.619 (US\$ 14,728) y $\$ 33^{\prime} 126.155$ (US\$ 16,442). No se encontraron diferencias estadísticamente significativas entre eventos adversos prevenibles y no prevenibles en ninguna de las categorías incluidas en el análisis de costos.

La ecuación resultante del modelo de costos obtenida mediante el análisis de regresión fue:

$C D=-547.930,345$ - 0,539PD + 0,405VE + 0,310CM,

donde: CD son los costos medios directos totales; PD, las pruebas diagnósticas; VE, las valoraciones especializadas adicionales, y CM, el costo medio de los medicamentos adicionales.

Para comprobar la validez del modelo, se probaron los supuestos de normalidad, "homo- sedasticidad", independencia de los residuos y no "multicolinealidad", mediante las pruebas correspondientes.

\section{Discusión}

Se encontró un porcentaje de eventos adversos prevenibles de 24,8 \%. No se observó una mayor tendencia a desarrollar eventos o reacciones adversas a medicamentos en los grupos de mayor edad, ni tampoco se encontraron diferencias según el sexo. En los casos estudiados, los mayores porcentajes de eventos prevenibles se asociaron a fármacos que actúan sobre la sangre y los órganos hematopoyéticos, y a los diuréticos.

Al evaluar la causalidad, se encontró un mayor porcentaje de eventos calificados como posibles $(43,1 \%)$, seguidos por los eventos dudosos $(26,6$ $\%$ ), mientras que $12,3 \%$ se clasificó como definitivo y, 16,7 \%, como probable. En lo referente a la gravedad, cerca de la mitad de los casos (49,8\%) no requirieron ninguna modificación en el tratamiento 
Cuadro 5. Costos directos asociados a eventos adversos prevenibles a medicamentos (en pesos colombianos)

\begin{tabular}{|c|c|c|c|c|c|}
\hline Intervenciones & $\mathbf{N}$ & $\begin{array}{l}\text { Costos } \\
\text { totales }\end{array}$ & $\begin{array}{c}\text { Casos de } \\
\text { prevenibles (\%) }\end{array}$ & $\begin{array}{c}\text { Costo } \\
\text { prevenibles }\end{array}$ & $\begin{array}{l}\text { Costo } \\
\text { total (\%) }\end{array}$ \\
\hline Pruebas diagnósticas & 203 & $\$ 18^{\prime} 777.700$ & 34,0 & $\$ 7 ’ 045.300$ & 37,5 \\
\hline Valoraciones especializadas & 91 & $\$ 6{ }^{\prime} 278.600$ & 35,2 & $\$ 2 ' 838.900$ & 45,2 \\
\hline Procedimientos adicionales & 55 & $\$ 27^{\prime} 203.171$ & 41,8 & $\$ 7 ’ 936.587$ & 29,2 \\
\hline Medicamentos adicionales & 131 & & 37,4 & & \\
\hline Máximo & & $\$ 21^{\prime} 024.048$ & & $\$ 7 ’ 902.168$ & 37,6 \\
\hline Mínimo & & $\$ 14^{\prime} 821.222$ & & $\$ 5 ' 639.862$ & 38,1 \\
\hline Medio & & $\$ 17 ’ 159.757$ & & $\$ 6{ }^{\prime} 573.214$ & 38,3 \\
\hline Institución & & $\$ 11^{\prime} 187.942$ & & $\$ 3 ’ 767.958$ & 33,7 \\
\hline $\begin{array}{l}\text { Estancia hospitalaria adicional } \\
\text { Costos directos totales }(n=283)\end{array}$ & 34 & $\$ 20 ’ 439.000$ & 55,9 & $\$ 12^{\prime} 031.900$ & 58,9 \\
\hline Máximo & & $\$ 93722.519$ & & $\$ 37754.855$ & 40,3 \\
\hline Mínimo & & $\$ 87 ’ 519.693$ & & $\$ 35 ’ 492.549$ & 40,6 \\
\hline Medio & & $\$ 89 ’ 858.228$ & & $\$ 36^{\prime} 425.901$ & 40,5 \\
\hline Institución & & $\$ 83^{\prime} 886.413$ & & $\$ 33 ’ 620.645$ & 40,1 \\
\hline
\end{tabular}

con el medicamento sospechoso ni ninguna acción adicional. En 25,9\% de los casos se requirió retiro o cambio del medicamento o tratamiento adicional, sin incrementar el tiempo de estancia hospitalaria. La mortalidad global asociada a eventos adversos fue de $2,5 \%$, mientras que la relacionada con eventos adversos prevenibles correspondió a 1,1 $\%$ del total. Respecto al carácter prevenible de los eventos revisados, se encontró que el mayor número de casos prevenibles se asoció a la dosis, ruta o frecuencia de administración de los medicamentos no apropiados para la edad, el peso o el estado de la enfermedad, y a la presencia de interacciones con medicamentos.

Los costos directos totales asociados a eventos adversos a medicamentos oscilaron entre \$ $83^{\prime} 886.413$ y $\$ 93$ '722.519 pesos, de los cuales se estima que entre 40,1 y $40,6 \%$ correspondían a casos prevenibles, \$33'620.645 (US\$16.687) y \$ 37’754.855 (US\$18.739), respectivamente.

El porcentaje de eventos prevenibles encontrado en este estudio (24,8\%), está acorde con lo publicado por Bates, et al., quienes encontraron un porcentaje de $24 \%$ en un hospital universitario (17). Sin embargo, la variabilidad entre los porcentajes es amplia en diferentes estudios que evalúan el porcentaje de eventos prevenibles (18,7 a 73,2 \%) (6). Del total de casos prevenibles, $46,8 \%$ tuvo una relacion de causalidad definitiva o probable.

Al analizar el subconjunto de reacciones adversas a medicamentos (aquellos eventos donde la relación de causalidad resultó definitiva o probable), este porcentaje de casos prevenibles sube a $40,3 \%$ entre los 129 casos analizados. Esto podría asociarse a que en las reacciones adversas a medicamentos, una vez establecida una mayor relación de causalidad, se tienen mayores elementos de juicio para establecer si son prevenibles o no.

El hecho de no encontrar una mayor tendencia a desarrollar eventos o reacciones adversas a medicamentos en los grupos de mayor edad, ni encontrar diferencias según el sexo, coincide con el hallazgo de Tribiño, et al., quienes realizaron un estudio en el Servicio de Medicina Interna de la misma institución (18). No obstante, varios autores señalan una mayor tendencia entre las mujeres a presentar reacciones adversas a los medicamentos, con respecto a los hombres $(19,20)$.

Dentro de los grupos de medicamentos más comúnmenterelacionadosconeventosadversos, se encuentra que los mayores porcentajes de eventos prevenibles se asociaron a fármacos que actúan sobre la sangre y los órganos hematopoyéticos $(32,3 \%)$, y los diuréticos (42,9\%). Estos dos grupos anatomo-terapéuticos se describen como factores de riesgo para la presentación de reacciones adversas (21).

En lo referente a la gravedad, tomando los fallecimientos asociados directa o indirectamente con eventos adversos, el porcentaje encontrado en este estudio (2,5\%) es similar al reportado por Davies, et al. (19).

Al explorar diferencias entre eventos prevenibles y no prevenibles, sólo en casos definitivos y probables, se encontró una diferencia estadísticamente significativa con una relación directa con su carácter prevenible en variables relacionadas con la gravedad del evento adverso 
a medicamentos (necesidad de procedimientos, pruebas diagnósticas adicionales, aumento en estancia hospitalaria y evento serio). Gholami y Shalviri encontraron que los casos prevenibles eran mas graves, se incrementaban con la edad del paciente y prolongaban más el tiempo de estancia, con respecto a los no prevenibles (22). En el presente estudio, se halló una diferencia significativa entre los eventos prevenibles y los no prevenibles, con respecto a eventos asociados a warfarina (63,8 \% casos prevenibles). Winterstein, et al., encontraron que la anticoagulación excesiva y los eventos hemorrágicos representaron un tercio del total de reacciones adversas prevenibles (23).

En el análisis general, se encontró que el mayor número de eventos prevenibles se asoció a dosis, ruta o frecuencia de administración de los medicamentos no apropiados para la edad, el peso o el estado de la enfermedad (51,4\%), y a interacciones medicamentosas (45\%), los cuales están descritos dentro de los problemas relacionados con medicamentos (24). En el estudio de Winterstein, et al., se encontró que 68,1 \% de las reacciones prevenibles se asoció con el primer factor mencionado, mientras que $26,6 \%$ se asoció con las interacciones entre medicamentos (23), siendo éstas las áreas que pueden intervenirse y en las cuales se obtendrían mejores resultados en términos de disminución de la presentación de eventos adversos.

En el aspecto económico, un porcentaje mayoritario de casos $(63,2 \%)$ requirió de intervenciones adicionales que se tradujeron en costos asociados. De estos, los componentes que generaron mayor costo fueron la estancia hospitalaria y los procedimientos adicionales. Este porcentaje de casos que generaron costos por diferentes conceptos, difiere del reportado por Tribiño, et al., (74,6 \%) (18). La diferencia se puede explicar porque el presente estudio incluyó reportes correspondientes a fallas terapéuticas atribuidas principalmente a analgésicos, que en muchas ocasiones no requirieron intervenciones adicionales que se tradujeran en costos directos.

Los costos directos totales asociados a eventos oscilaron entre \$83'886.413 (US\$ 41.636) y \$93'722.519 (US\$ 46.518), de los cuales se estima que entre 40,1 y 40,6 \% (\$33'620.645 y $\$ 37$ '754.855, respectivamente), se atribuyeron a casos prevenibles, sin mostrar diferencia significativa respecto a los costos generados por eventos no prevenibles, ni tampoco al comparar cada una de las categorías en las que se agruparon los costos. Sin embargo, al analizar únicamente las reacciones adversas definitivas o probables, esta diferencia sí resulta significativa, pudiéndose afirmar que las reacciones adversas prevenibles consumen mayores recursos que las no prevenibles, lo cual está acorde con lo descrito en la literatura científica $(3,25,26)$.

Si bien el diseño de este estudio fue planteado como de tipo descriptivo, teniendo en cuenta la cantidad de información recolectada, se decidió aprovecharla para plantear un modelo predictor de costos directos asociados, el cual muestra que, para los eventos prevenibles, los principales componentes que permiten pronosticar los costos medios directos asociados a los casos estudiados son los costos totales por pruebas diagnósticas, por medicamentos y por valoraciones especializadas, lo cual ocasiona un incremento en el uso del recurso en salud, que en un momento dado puede ser limitado en la institución.

La evaluación desde el punto de vista de la prevención, obliga a examinar los servicios clínicos y a revisar el nivel del cuidado farmacéutico con el que cuenta la institución, de manera que la evaluación de reacciones adversas a medicamentos por gravedad, tipo de reacción y tipo de medicamento, brinde un mecanismo para el mejoramiento de la calidad en la farmacia y al comité médico para identificar oportunidades de intervención como fuente de ahorro de recursos en el sistema de salud (14,26). Cabe anotar que son pocos los estudios que evalúan el impacto económico de la presentación de eventos y reacciones adversas a medicamentos en el contexto local, y es la primera vez que se efectúa un análisis utilizando una herramienta que permite discriminar el carácter prevenible de los eventos, identificando cuáles son los principales factores asociados sobre los cuales se puede intervenir y, de esta manera, mejorar la calidad de la atención de los pacientes, lo cual permite un mejor aprovechamiento de los recursos en salud.

Entre las limitaciones de este estudio, y dadas las características propias del hospital donde se realizó, los resultados pueden no ser aplicables a otra institución de salud. Por otra parte, los análisis se hicieron de manera retrospectiva sobre los datos contenidos en la historia clínica, de manera que, al no tener interacción con los médicos tratantes y pacientes, no hay posibilidad de indagar más a fondo en ciertos casos y así tener mayores elementos de juicio a la hora de evaluar 
la causalidad y el carácter prevenible. De la misma forma, al incluir únicamente tres servicios, pudo presentarse una dilución de los datos, por lo que se recomienda que, a la hora de implementar tareas de farmacovigilancia, los esfuerzos se enfoquen específicamente por servicio, grupos etarios de pacientes y medicamentos.

En el presente estudio se encontró que los eventos prevenibles se asocian principalmente a interacciones entre medicamentos y a esquemas de dosificación inadecuados, y se relacionan con mayores costos de atención, lo cual se estableció mediante la evaluación de su carácter prevenible mediante los criterios de Schumock y Thornton. Por esta razón, la implementación de esta evaluación en laprácticaclínicahabitual podría seruna herramienta útil para identificar y prevenir la presentación de reacciones adversas a medicamentos. Por otra parte, se sugiere implementar, en las instituciones de salud, actividades pedagógicas resaltando el uso racional de medicamentos, enfocadas a posibles repercusiones sobre los pacientes, de esquemas de dosificación o combinaciones de medicamentos inadecuados.

\section{Agradecimientos}

Los autores expresan su gratitud al personal de las áreas de Estadística, Sistemas y de la Dirección de Educación Médica del Hospital Universitario La Samaritana de Bogotá, que colaboraron con esta iniciativa y que brindaron su apoyo para hacer posible su realización.

\section{Conflicto de intereses}

Los autores declaran que no tienen conflictos de intereses.

\section{Financiación}

Esta investigación fue realizada como parte de los requisitos para optar al título de Magíster en Farmacología de la Universidad Nacional de Colombia. Como tal, la investigación no contó con financiación de fuente específica (beca o subvención).

\section{Referencias}

1. Schumock GT, Thornton JP. Focusing on the preventability of adverse drug reactions. Hosp Pharm. 1992;27:538.

2. Brennan TA, Leape LL, Laird NM, Hebert L, Localio $\mathbf{R}$, Lwthers AG, et al. Incidence of adverse events and negligence in hospitalized patients. Results of the Harvard Medical Practice Study. N Engl J Med. 1991;324:370-6.

3. Gautier S, Bachelet H, Bordet R, Caron J. The cost of adverse drug reactions. Expert Opin Pharmacother. 2003;4:319-26.
4. Temple ME, Robinson RF, Miller JC, Hayes JR, Nahata MC. Frequency and preventability of adverse drug reactions in paediatric patients. Drug Saf. 2004;27:819-29.

5. van den Bemt PM, Egberts TC, de Jong-van den Berg LT, Brouwers JR. Drug-related problems in hospitalised patients. Drug Saf. 2000;22:321-33.

6. Kanjanarat P, Winterstein AG, Johns TE, Hatton RC, González-Rothi R, Segal R. Nature of preventable adverse drug events in hospitals: A literature review. Am. J HealthSyst Pharm. 2003;60:1750-9.

7. Nebecker JR, Barach $\mathbf{P}$, Samore MH. Clarifying adverse drug events:Aclinician's guide to terminology, documentation and reporting. Ann Intern Med. 2004;140:795-801.

8. Johnson JA, Bootman JL. Drug-related morbility and mortality: A cost of illness model. Arch Int Med. 1995;155:1949-56.

9. Montoya G. Programa de detección de eventos adversos asociados a la administración de tramadol y dipirona en un hospital de alta complejidad. (trabajo de grado). Bogotá, D.C.: Universidad Nacional de Colombia; 2008. p. 46.

10. Parra MF. Programa de detección de eventos adversos asociados a la administración de heparina y warfarina en un hospital de alta complejidad (trabajo de grado). Bogotá, D.C.: Universidad Nacional de Colombia; 2008. p. 79.

11. Rehan HS, Chopra D, Kakkar AK. Physician's guide to pharmacovigilance: Terminology and causality assessment. Eur J Intern Med. 2009;20:3-8.

12. Committee of Experts on Management of Safety and Quality in Health Care (SP-SQS). Expert Group on Safe Medication Practices. 2005. Glossary of terms related to patient and medication safety. Genova: World Health Organization; 2005. Fecha de consulta: 14 de enero de 2010. Disponible en: http://www.who.int/patientsafety/ highlights/COE_patient_and_medication_safety_gl.pdf.

13. Uppsala Monitoring Centre. The use of the WHO-UMC system for standardized case causality assessment. Genova: World Health Organization. Fecha de consulta: 14 de enero de 2010. Disponible en: www.who-umc.org/ graphics/4409.pdf.

14. Hartwig S, Siegel J, Schneider P. Preventability and severity assessment in reporting adverse drug reactions. Am J Hosp Pharm. 1992;49:229-32.

15. Uppsala Monitoring Centre. Glossary of terms used in pharmacovigilance. Genova: World Health Organization; 2006. Fecha de consultado: 14 de enero de 2010. Disponible en: http://www.who-umc.org/graphics/15338.pdf.

16. Banco de la República de Colombia. Series estadísticas. 2007 Fecha de consulta: 17 de abril de 2008. Disponible en: http://www.banrep.gov.co/series-estadisticas/see_ts_cam. $\mathrm{htm}$.

17. Bates DW, Miller EB, Cullen DJ, Burdick L, Williams L, Laird N, et al. Patient risk factors for adverse drug events in hospitalized patients. Arch Intern Med. 1999;159:2533-60.

18. Tribiño G, Maldonado C, Segura O, Díaz J. Costos directos y aspectos clínicos de las reacciones adversas a medicamentos en pacientes hospitalizados en el servicio de medicina interna de una institución de tercer nivel de Bogotá. Biomédica. 2006;26:31-41. 
19. Davies EC, Green CF, Taylor S, Williamson PR, Mottram DR, Pirmohamed M. Adverse drug reactions in hospital inpatients: A prospective analysis of 3,695 patient-episodes. PLoS One. 2009;4:e4439.

20. Ajayi F, Sun H, Perry J. Adverse drug reactions: A review of relevant factors. J Clin Pharmacol. 2000;40:1093-101.

21. Krahenbuhl-Melcher A, Schlienger R,Lampert $\mathbf{M}$, Haschke M, Dreive J, Krahenbuhd S. Drug-related problems in hospitals. A review of the recent literature. Drug Saf. 2007;30:379-407.

22. Gholami K, Shalviri G. Factors associated with preventability, predictability, and severity of adverse drug reactions. Ann Pharmacother. 1999;33:236-40.

23. Winterstein AG, Hatton RC, González-Rothi R, Johns TE, Segal R. Identifying clinically significant preventable adverse drug events through a hospital's database of adverse drug reaction reports. Am J Health Syst Pharm. 2002;59:1742-9.

24. Comité de Consenso. Tercer Consenso de Granada sobre problemas relacionados con medicamentos (PRM) y resultados negativos asociados a la medicación (RNM). Ars Pharm. 2007;48:5-17

25. Bates DW, Spell N, Cullen DJ, Burdick E, Laird N, Petersen LA, et al. The costs of adverse drug events in hospitalized patients. JAMA. 1997;277:307-31.

26. Lagnaoui F, Moore N, Fach L, Longy-Boursier M, Begaud B. Adverse drug reactions in a department of systemic diseases-oriented internal medicine: Prevalence, incidence, direct costs and avoidability. Eur J Clin Pharmacol. 2000;55:181-6. 